\title{
onerers
}

\section{Closing the NET on impaired wound healing in diabetes mellitus}

$\mathrm{N}$ eutrophil extracellular traps (NETs) are formed in response to microbial infections and are composed of extracellular networks of decondensed chromatin coated with cytotoxic proteins and histones to eliminate the pathogen. However, NETs are also associated with tissue damage and can induce inflammatory and autoimmune disorders. In patients with diabetes mellitus, wound healing is impaired, and raised glucose levels have been suggested to produce an environment that is conducive to increased formation of NETs, or 'NETosis'. In new data presented by investigators from the USA, diabetic conditions do indeed seem to promote NETosis, which results in poorer wound healing than in normoglycaemia. However, this effect might be countered by treatments that degrade secreted chromatin.

"We found that NETs hindered wound healing even under normoglycaemic conditions," explains study author Denisa Wagner. "As healing is a major problem in diabetes mellitus we asked whether NETs form more prominently in patients with diabetes mellitus than in those with normoglycaemcia and if this process could delay healing."

Using neuotrophils from blood samples stimulated with ionomycin, which increases intracellular calcium, patients with diabetes mellitus were found to produce more NETs than healthy normoglycaemic individuals. Levels of PAD4, a calcium-dependent protein that modifies histones by converting an arginine to citrulline and facilitates the chromatin decondensation in NETosis, were also increased in patients with diabetes mellitus. Similarly, mice treated with streptozotocin to induce diabetes mellitus and $d b / d b$ mice both had an increased proportion of neutrophils that were positive for citrullinated histone $\mathrm{H} 3$ compared with normoglycaemic control mice. Taken together, these data suggest that NETosis is enhanced in diabetic conditions, regardless of type or origin.

Given the association between diabetes mellitus and impaired wound healing, and that NETosis can induce epithelial cell death, the team next investigated the role of NETs in wound repair. "Because neutrophil behavior can be modified by hyperglycaemia and diabetic conditions, we [want to] expand our knowledge of diabetic neutrophil biology and how neutrophils and NETs have a role in diabetic complications," explains coauthor Siu Ling Wong.

After establishing that neutrophils are the main source of citrullinated histone $\mathrm{H} 3$ in skin wounds, the team used a PAD4 knockout mouse line (Padi4 $\left.{ }^{-/-}\right)$to further investigate the role of NETs in wound healing. Skin wounds in Padi4 ${ }^{-/-}$mice healed faster, and re-epithelialization was quicker, than in wild-type mice. Interestingly, wound healing was not impaired in streptozotocin-treated Padi4 $^{-/-}$mice, which supports the hypothesis that NETosis can delay healing in diabetic conditions.

As PAD4 is not expressed in the skin, the team hypothesized that the impaired wound healing was the result of the infiltrating neurophils producing NETs. Consequently, digesting the NETs with DNAse 1, which might speed up removal of NETs by macrophages, might also enhance wound repair. Indeed, in diabetic mice, DNAse 1 treatment reduced wound size and improved re-epithelialization, which was similar to the speed and extent of wound healing in DNAse 1-treated normoglycaemic mice. Similarly, wound healing was improved with DNAse 1 in normoglycaemic mice compared with those treated with a control compound. This effect was not seen in $\mathrm{Padi4}^{-/-}$mice, which provides further supporting evidence that formation of NETs produces extracellular DNA that is detrimental to wound healing.

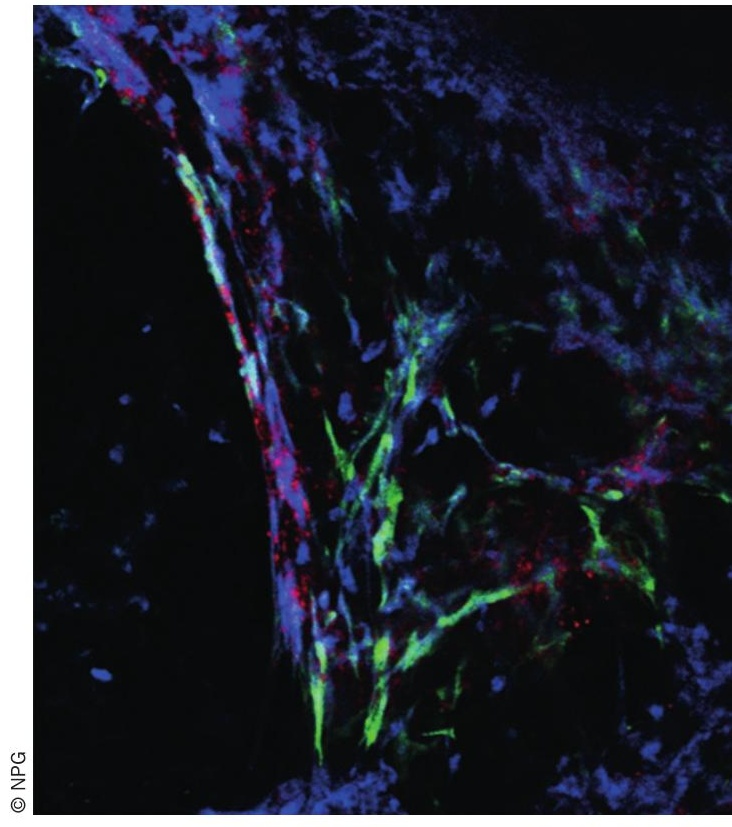

Taken together, these results suggest that a DNAse 1-based treatment could be used to enhance wound healing in patients. "Our future studies will hopefully provide significant and innovative steps towards both preclinical understanding of NETs and clinical treatments of diabetic wounds." explains Wong. "The goal of [all] diabetes research is to prevent the occurrence of diabetes mellitus and improve the quality of life for patients." However, these findings have wider implications than just those in the context of diabetes mellitus. "We would like to know exactly how NETs interfere with wound closure and what will be the consequences of excessive or the absence of NETosis on scar formation, we hope to work with a clinicians to test inhibition of NETs in human wound healing," explains Wagner.

\section{Tim Geach}

Original article Wong, S. L. et al. Diabetes primes neutrophils to undergo NETosis, which impairs wound healing. Nat. Med. doi:10.1038/nm.3887 